\title{
EL CUESTIONARIO CEPEA: HERRAMIENTA DE EVALUACIÓN DE ENFOQUES DE APRENDIZAJE EN ESTUDIANTES UNIVERSITARIOS ${ }^{1}$
}

\author{
Raquel Salim \\ Universidad Nacional de Tucumán, Argentina
}

\section{INTRODUCCIÓN}

En el momento actual cada vez cobra mayor importancia el estudio del aprendizaje desde la perspectiva del alumno, que es quien otorga significado y sentido a los materiales que procesa y decide lo que tiene que aprender, así como la manera de hacerlo. Nuestro interés se centra en conocer los procesos que utiliza para aprender.

Parece aceptado que aprender requiere disposición y utilización de las estrategias precisas. Esta disposición implica necesariamente motivación (o más concretamente un conjunto de variables como las metas de aprendizaje y el autoconcepto), pero también qué concepción de aprendizaje mantiene el estudiante y cómo lo aborda (enfoques de aprendizaje).

Estas cuestiones, relacionadas entre sí, constituyen el tema que abordamos en esta investigación: los tipos de enfoques y estrategias de aprendizaje que utilizan los estudiantes universitarios y cómo se relacionan éstos con sus actividades de aprendizaje para mostrar un perfil de la población seleccionada en relación con sus expectativas de éxito.

Las diferencias en la forma en que los estudiantes abordan las situaciones de aprendizaje propició el surgimiento de una corriente investigadora, desarrollada en distintas universidades del mundo, interesada en el estudio de los enfoques de aprendizaje. Estas investigaciones han tenido gran resonancia en el ámbito de la investigación educativa por la relación que establecen entre aspectos estratégicos y motivacionales del aprendizaje.

Se observó una notable consistencia en la identificación de dos tipos de enfoques de aprendizaje: superficial (orientación hacia la reproducción) y profundo (orientación hacia la comprensión del significado). Swenson en 1977 demostró que los estudiantes que adoptan un estilo profundo, emplean más tiempo en el estudio y consideran el material que aprenden como más fácil de comprender que los estudiantes que adoptan un estilo superficial. Los segundos, al concentrarse en estrategias de memorización y retención, encuentran el trabajo pesado e ingrato, y suelen fracasar más frecuentemente en los exámenes. También se ha analizado (Fransson 1977) que si la situación de aprendizaje está muy orientada al rendimiento y a la evaluación de resultados, los estudiantes pueden adoptar estilos superficiales que les conducen a intentar memorizar la información para superar las pruebas de evaluación o los exámenes.

Algunos autores coinciden en asociar un tipo de motivación con cada uno de los enfoques de aprendizaje. Han constatado que existe una cierta relación entre el tipo de motivación y los enfoques de

\footnotetext{
${ }^{1}$ Ponencia presentada en el IV Encuentro Nacional y I Latinoamericano 'La Universidad como Objeto de Investigación' - Tucumán, R. Argentina - Octubre 2004. Resultados preliminares del proyecto de tesis de Maestría en Docencia Superior Universitaria: "Enfoques y Estrategias de Aprendizajes en Estudiantes Universitarios. Estudio de un caso.
} 
aprendizaje que manifiestan los alumnos ante una determinada tarea de aprendizaje. Así, el tipo de motivación predominante en el individuo podría estar significativamente relacionado con la utilización de determinadas estrategias de aprendizaje y, consiguientemente, con el enfoque usado por el alumno (Biggs 1985, 1989, 1991; Entwistle 1987; Schmeck 1988; Weinstein, Goetz y Alexander 1988).

Las relaciones motivo-estrategia en las que se basan los enfoques de aprendizaje pueden describirse en los siguientes términos: aquellos estudiantes que tienen la intención de cumplir los requisitos mínimos de la tarea, con un mínimo de esfuerzo e implicación en la misma, pondrán en marcha determinadas estrategias dirigidas a aprender mecánica y repetitivamente la información y reproducirla en el momento oportuno. Estas relaciones motivo-estrategia reflejan las características del enfoque superficial. Por otro lado, aquellos estudiantes con un alto interés intrínseco y un alto grado de implicación en lo que están aprendiendo, con la intención de comprenderlo significativa mente desarrollarán estrategias dirigidas a descubrir el significado de lo que van a aprender estableciendo relaciones con conocimientos previos relevantes. Estas relaciones motivo-estrategia reflejan las características del enfoque profundo (Valle Arias, González Cabanach 1997).

Pero además de los dos enfoques mencionados, se ha identificado un tercero denominado 'enfoque de logro' (Biggs 1988a, Entwistle 1988) que implica una intención claramente definida; obtener el máximo rendimiento posible a través de una planificación adecuada de las actividades, del esfuerzo y del tiempo disponible. Es decir que este enfoque se caracteriza por la planificación y organización de las distintas actividades con el objetivo prioritario de obtener logros académicos lo más altos posibles (Valley col. 1993).

Se puede considerar que mientras el enfoque profundo y el superficial son, en cierta medida, excluyentes, el enfoque de logro puede vincularse a una aproximación profunda o superficial dependiendo del contexto particular de aprendizaje. Dentro de estos factores contextuales existe un acuerdo en señalar el importante papel que desempeñan los criterios de evaluación en la adopción de un enfoque de logro (motivo o intención de obtener altas calificaciones), combinado con un enfoque profundo o superficial. Como sugieren González Cabanach y col. (1993), aquellos alumnos que creen que la mejor manera de conseguir altas calificaciones consiste en aprender mecánica y repetitivamente el material de aprendizaje, sin necesidad de implicarse en la comprensión y significatividad del mismo, posiblemente combinen los enfoques superficial y de logro. Por el contrario, los alumnos que consideran que la obtención de altas calificaciones depende de la comprensión y de las relaciones que se establezcan entre el nuevo aprendizaje y los conocimientos previos, es posible que adopten una combinación de los enfoques profundo y de logro. La combinación de enfoques da lugar a los 'enfoques compuestos'.

\section{MATERIALES Y MÉTODOS}

Para evaluar el grado y rivel de los enfoques de aprendizaje que adopta un estudiante de nivel universitario en su proceso de estudio, así como los motivos y estrategias más relevantes que integran dichos enfoques de aprendizaje y los compuestos de enfoques utilizamos el Cuestionario de Evaluación de Procesos de Estudio y Aprendizaje para el alumnado universitario (CEPEA) diseñado por Alfonso Barca Lozano (1999). El cuestionario se cumplimenta en una escala tipo Likert (1-5) compuesto por 42 items que proporcionan la obtención de puntuaciones para 6 subescalas: 3 de Motivos (superficial, profunda y logro) y 3 de Estrategias de aprendizaje (superficial, profundo y logro) que implican las tareas de estudio y aprendizaje en general. En un segundo nivel, se obtienen las puntuaciones de 3 Escalas de Enfoques de 
Aprendizaje que integran a los motivos y estrategias y, por último, los dos Compuestos de Enfoques (superficial-logro y profundo-logro). Es de aplicación individual y el tiempo de aplicación es variable, entre 15 y 20 minutos.

Los estudiantes, de forma voluntaria y en el aula donde habitualmente recibían sus clases llenaron y entregaron el cuestionario. Una vez cumplimentado el Cuestionario se realizó su análisis y evaluación. Se obtuvo un perfil prototípico de las mediciones de as distintas sub-escalas y escalas en sus diferentes categorías.

La clasificación de grupos, lo más homogéneos posibles se realizó mediante el análisis multivariado 'cluster' que permite clasificar grupos similares de individuos o variables que se van agrupando en conglomerados sin que respondan a una idea preconcebida. En la realización de los análisis estadísticos se utilizó el paquete estadístico SPSS para Windows versión 10.0 .

Población: Estaba conformada por 248 estudiantes universitarios, de ambos sexos, cuyas edades oscilaban entre los 20 y 27 años. De ellos, 86 (34,7\%) alumnos cursaban la asignatura Biología (2ำ año-ciclo básico), 67 (27,0\%) Microbiología General (4- año-ciclo intermedio) y 95 (38,3\%) Micología (5º año-ciclo profesional).

Muestra: Por tratarse de una metodología observacional que no se propone una explicación causal sino que se propone comprender los significados que atribuyen los estudiantes al aprendizaje, para las determinaciones cuantitativas se trabajó con una muestra no aleatoria compuesta por estudiantes voluntarios.

\section{RESULTADOS}

De acuerdo a las características del cuestionario es posible identificar cuatro variables (motivaciones, estrategias, enfoques y compuestos) que adoptan tres categorías (superficial, profundo y de logro).

\subsection{Los Enfoques}

Gráfico 1.

Cluster de Enfoques

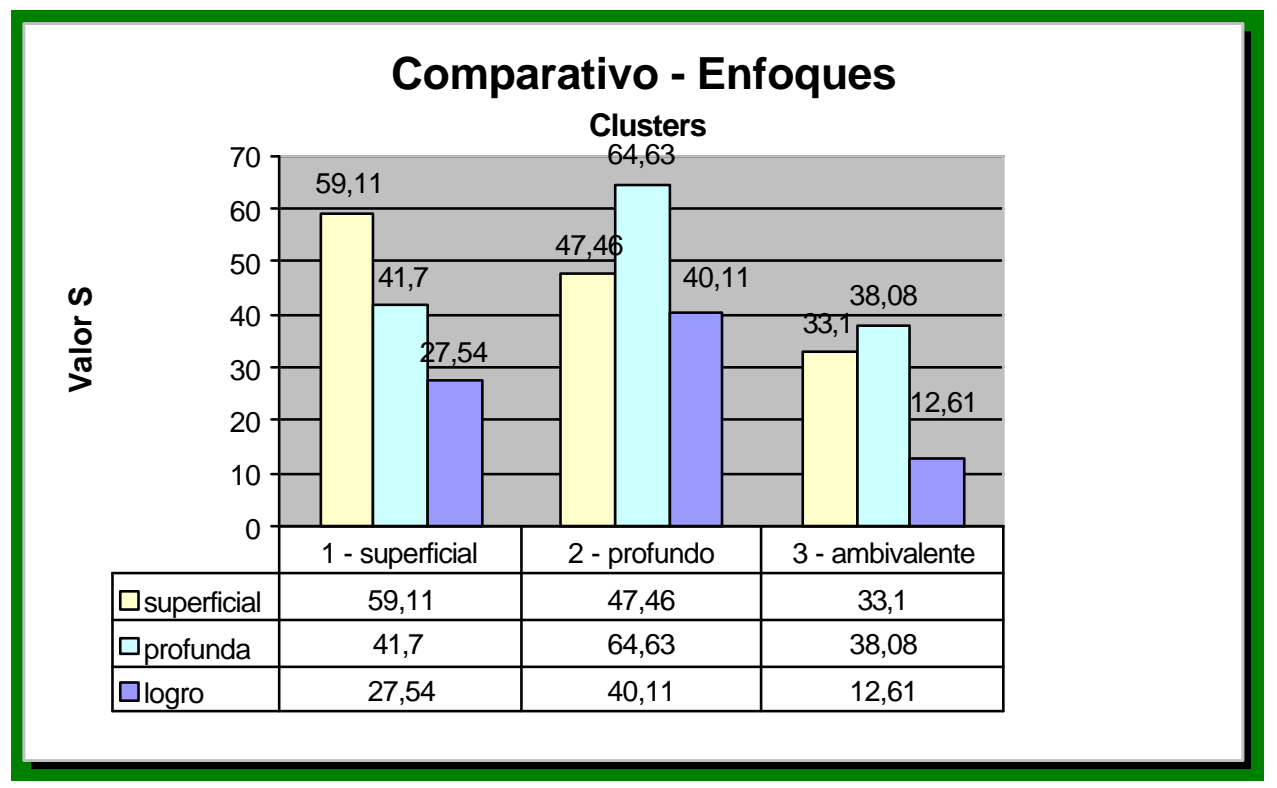


Los resultados del estudio identificaron 3 'clusters' o grupos de estudiantes que presentan diferencias importantes en los enfoques de aprendizaje -motivos y estrategias- que utilizan cuando se enfrentan a las tareas académicas propias de la educación universitaria.

Estos resultados nos proporcionan indicios de la existencia de dos grupos de estudiantes universitarios (cluster 1 y cluster 2) que se diferencian por el tipo de enfoque de aprendizaje que utilizan preponderantemente cuando se enfrentan a los aprendizajes habituales de este nivel educativo.

Por un lado, un grupo de 56 alumnos, que tienen en común el adoptar un enfoque superficial con un componente de motivo superficial (cluster 1), y otro grupo (cluster 2), más numeroso de alumnos (139) que adoptan predominantemente un enfoque profundo con un componente de estrategia profundo y de logro. Lo que resulta sorprendente es la identificación de un grupo de alumnos (53) que no coinciden en ninguna de las dos categorías de enfoques mencionados anteriormente (cluster 3). Este grupo de estudiantes -'grupo ambivalente'- no adoptan destacadamente ninguno de los dos enfoques prototípicos.

Distribución de la Población encuestada: La Tabla 1 muestra que del total de alumnos encuestados $139(56,0 \%)$ se ubican en el cluster 2 enfoque profundo-. La distribución del resto de la población en los cluster $1(22,6 \%)$ y cluster $3(21,4 \%)$ no muestra diferencias significativas. Más de la mitad de la población encuestada se ubica en el cluster 2 (profundo). Es interesante ver que a medida que se avanza en la carrera crece la población muestral del cluster 2, lo que indicaría un tránsito en el estilo de aprendizaje de superficial a profundo. Esto podría explicarse como un proceso de mayor adaptación al ambiente universitario (aprende el 'oficio' de estudiante universitario) y se acomoda mejor a las reglas. Un segundo aspecto puede encontrarse en su bagaje de conocimientos (conoce más, aprende mejor).

Tabla 1.

Distribución de la población estudiantil indagada para cada cluster.

\begin{tabular}{|l|c|c|c|c|c|c|c|c|}
\hline \multirow{2}{*}{ CURSO } & \multicolumn{2}{|c|}{ Cluster 1 } & \multicolumn{2}{c|}{ Cluster 2 } & \multicolumn{2}{c|}{ Cluster 3 } & \multicolumn{2}{c|}{ TOTAL } \\
\cline { 2 - 9 } & cantidad & $\%$ & cantidad & $\%$ & cantidad & $\%$ & cantidad & $\%$ \\
\hline & & & & & & & & \\
2do Biología & 25 & 29,1 & 44 & 51,2 & 17 & 19,8 & $\mathbf{8 6}$ & $\mathbf{1 0 0 , 0}$ \\
4to M. Gral & 14 & 20,9 & 37 & 55,2 & 16 & 23,9 & $\mathbf{6 7}$ & $\mathbf{1 0 0 , 0}$ \\
5to Micología & 17 & 17,9 & 58 & 61,1 & 20 & 21,1 & $\mathbf{9 5}$ & $\mathbf{1 0 0 , 0}$ \\
\hline \multicolumn{1}{|c|}{ TOTAL } & $\mathbf{5 6}$ & $\mathbf{2 2 , 6}$ & $\mathbf{1 3 9}$ & $\mathbf{5 6 , 0}$ & $\mathbf{5 3}$ & $\mathbf{2 1 , 4}$ & $\mathbf{2 4 8}$ & $\mathbf{1 0 0 , 0}$ \\
\hline
\end{tabular}

La Tabla 2 muestra que en el cluster 1 -enfoque superficial- predominan los alumnos que cursan el $2^{\circ}$ año de la carrera $(44,6 \%)$. En el cluster 2 -enfoque profundo- se encuentra un franco predominio de alumnos de $5^{\circ}$ año $(41,7 \%)$ seguido de los alumnos de $2^{\circ}$ año $(31,7 \%)$. En el cluster 3 -'grupo ambivalente'se ubica el mayor porcentaje de la población que cursa $4^{\circ}$ año $(30,2 \%)$, con una clara mayoría de los alumnos de $5^{\circ}$ año $(37,7 \%)$.

Tabla 2.

Población por curso para cada cluster. Evolución por cluster.

\begin{tabular}{|l|c|c|c|c|c|c|c|c|}
\hline \multirow{2}{*}{ CURSO } & \multicolumn{2}{|c|}{ Cluster 1 } & \multicolumn{2}{c|}{ Cluster 2 } & \multicolumn{2}{c|}{ Cluster 3 } & \multicolumn{2}{c|}{ TOTAL } \\
\cline { 2 - 9 } & cantidad & $\%$ & cantidad & $\%$ & cantidad & $\%$ & cantidad & $\%$ \\
\hline 2do Biología & 25 & $\mathbf{4 4 , 6}$ & 44 & 31,7 & 17 & 32,1 & $\mathbf{8 6}$ & $\mathbf{3 4 , 7}$ \\
4to M. Gral & 14 & 25,0 & 37 & 26,6 & 16 & 30,2 & $\mathbf{6 7}$ & $\mathbf{2 7 , 0}$ \\
5to Micología & 17 & 30,4 & 58 & $\mathbf{4 1 , 7}$ & 20 & $\mathbf{3 7 , 7}$ & $\mathbf{9 5}$ & $\mathbf{3 8 , 3}$ \\
\hline \multicolumn{1}{|c|}{ TOTAL } & $\mathbf{5 6}$ & $\mathbf{1 0 0 , 0}$ & $\mathbf{1 3 9}$ & $\mathbf{1 0 0 , 0}$ & $\mathbf{5 3}$ & $\mathbf{1 0 0 , 0}$ & $\mathbf{2 4 8}$ & $\mathbf{1 0 0 , 0}$ \\
\hline
\end{tabular}




\subsection{La Motivación}

El análisis de los aspectos motivacionales se realiza en base a las respuestas de los estudiantes a los 21 ítems correspondientes del cuestionario. Como se mencionó, estos ítems están agrupados en tres categorías -superficial, profunda y de logro- que evalúan aspectos motivacionales diferentes. La Tabla anexa al gráfico presenta algunos datos estadísticos correspondientes a cada una de las categorías mencionadas.

Gráfico 2.

Comparativo de Motivación por Cluster.

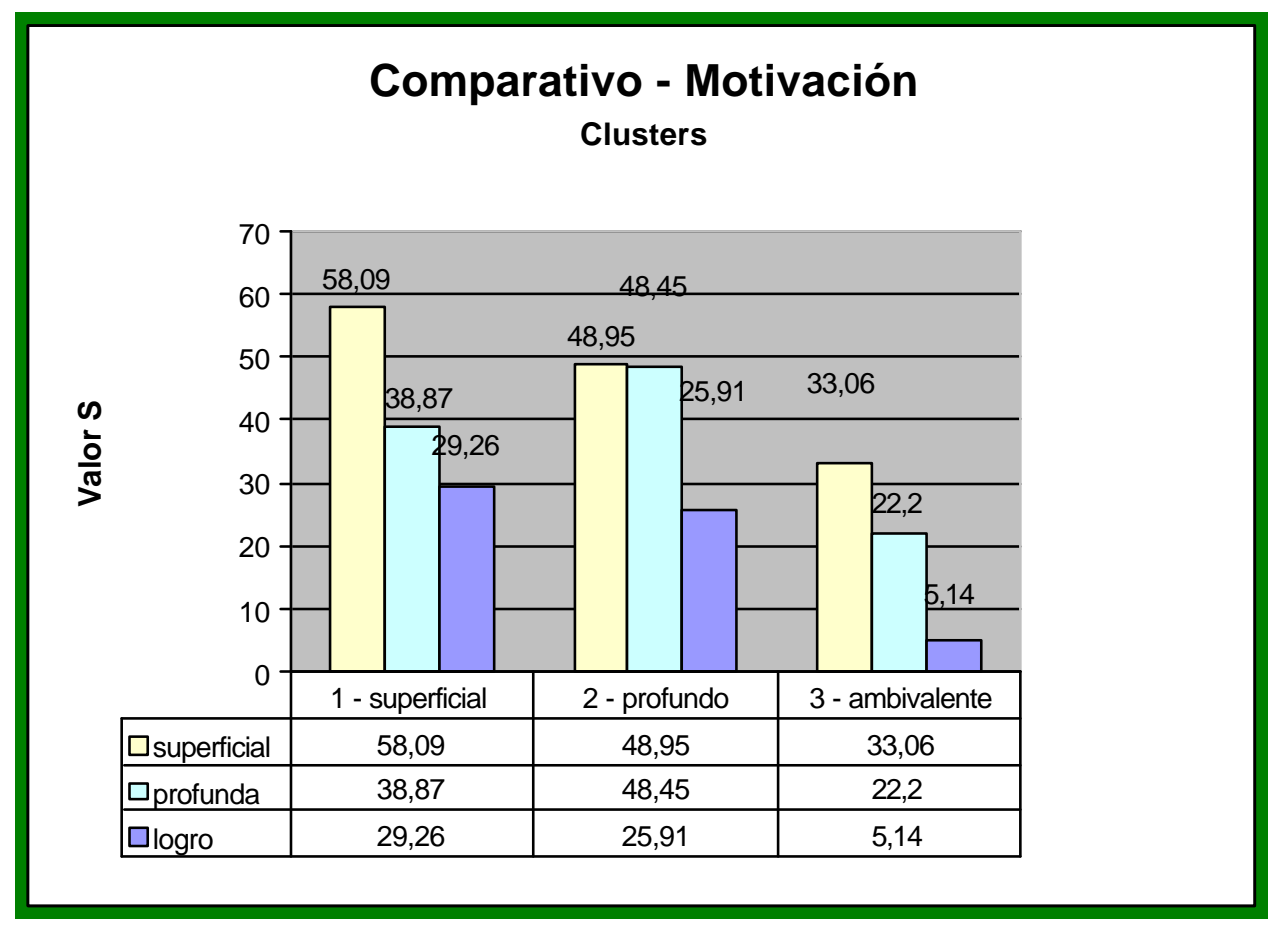

Estos datos permiten observar algunas características del perfil motivacional del grupo de estudiantes participantes. Se observa predominancia clara de la categoría superficial en el cluster superficial. Para el cluster profundo no se ven diferencias significativas entre las categorías superficial y profunda, por lo cual sería imposible identificar este cluster en términos de la motivación; esto podría deberse a limitaciones en la escala del instrumento en la medición de la motivación, ya que pueden observarse valores bastante bajos en comparación a las mediciones de estrategias. Lo anteriormente mencionado se observa claramente en el cluster 3 , en el que las mediciones son bajas con un predominio de la motivación superficial $(33,06)$.

\subsection{Las Estrategias}

El análisis de las estrategias se realiza en base a las respuestas de los estudiantes de los 21 ítems del cuestionario de la sección que evalúa las estrategias. También estos ítems están agrupados en tres categorías que evalúan el uso de estrategias diferentes por parte de los estudiantes. La Tabla presenta los valores de algunos índices estadísticos correspondientes a cada una de las categorías: superficiales, profundas y de logro. La consideración conjunta de las mismas permite una apreciación general acerca del uso de estrategias por parte de los estudiantes. 
Gráfico 3.

Comparativo de Estrategias por cluster.

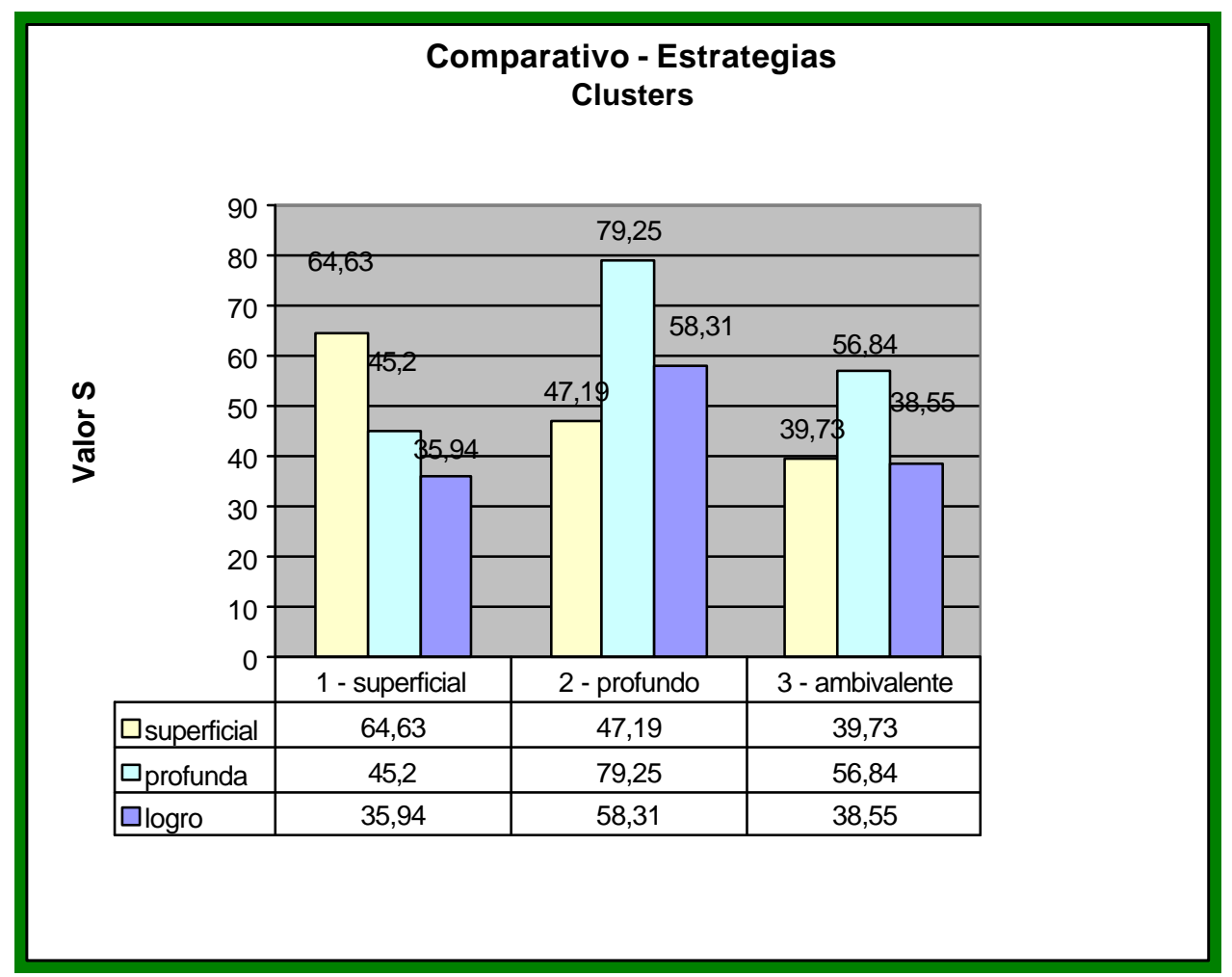

En este gráfico pueden identificarse claramente los clusters superficial y profundo con una resolución importante y diferencias estadísticas manifiestamente significativas. Esto mostraría cierto éxito del instrumento en la medición de esta variable. Para el cluster 3 -grupo ambivalente- se observa un componente bastante alto de estrategia profunda.

Al cruzar los valores de las variables relativas a la motivación y al uso de estrategias se puede advertir que no siempre los alumnos que tienen niveles de motivación más altos (gráfico 2) también hacen uso de las estrategias de mayor complejidad (gráfico 3) y, los sujetos con motivación baja tienden a recurrir en mayor medida al uso de estrategias de aprendizaje. Es decir que si bien estos alumnos no están demasiado motivados, igualmente ponen en marcha diversas estrategias cuando aprenden. Coincidentemente con nuestros resultados, Barca y col. (1999) encontraron igualmente que las subescalas estratégicas presentan índices más altos que las motivacionales.

\section{$3.4 \quad$ Los Compuestos}

Los compuestos son los valores medios de los enfoques en las categorías correspondientes; por ejemplo el compuesto profundo-logro (C-PL) resulta de promediar el enfoque profundo y el enfoque de logro.

El gráfico de compuestos muestra visiblemente definido el cluster 2 en términos de una medición alta y significativa de la categoría profunda (48,8 para el cluster 2). Sin embargo no es posible diferenciar entre clusters 1 y 2 en función de la medición de la categoría superficial (43,98 para cluster 1 vs. 42,2 para cluster 2). Esto puede deberse a las bajas mediciones de la categoría logro en general que proporciona el instrumento. 
Gráfico 4.

Comparativo de Compuestos por cluster.

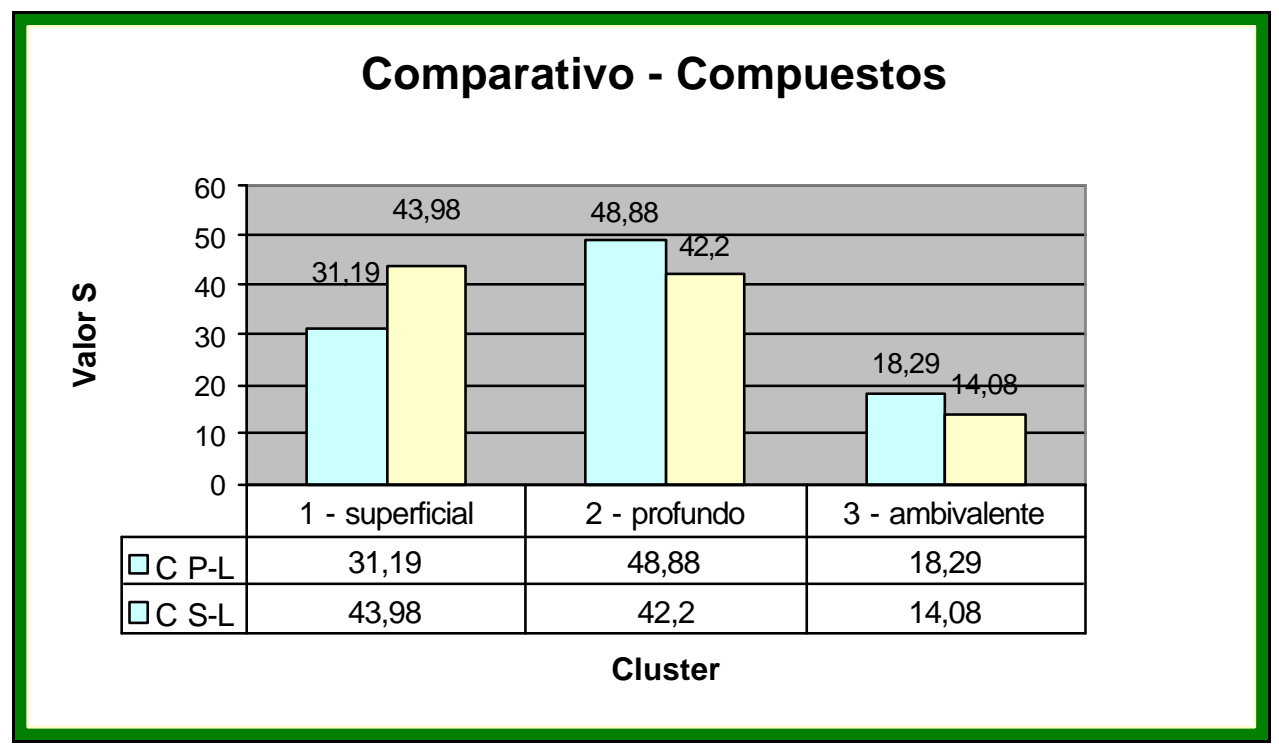

\section{CONCLUSIONES}

Mediante el procedimiento estadístico de clustering, hemos identificado 3 grupos de estudiantes que presentan diferencias importantes en los enfoques de aprendizaje -motivos y estrategias- que utilizan cuando se enfrentan a las tareas académicas propias de la educación universitaria: un grupo de alumnos $(22,6 \%)$ que tienen en común el adoptar un enfoque superficial con un componente de motivo superficial y otro grupo más numeroso $(56,0 \%)$ que adoptan un enfoque profundo con un componente importante de logro. Lo que resulta sorprendente es la identificación de un grupo $(21,4 \%)$ que no coincide con ninguna de las dos categorías de enfoques mencionados que hemos denominado 'grupo ambivalente'. Pareciera que este grupo de estudiantes se enfrenta a las actividades académicas sin motivos ni estrategias claras para su resolución.Las características que pueden definir a este grupo, probablemente tienen correspondencia con las 'metas de evitación del trabajo' que representan una forma de 'evitar la motivación'. En este sentido, es el grupo ambivalente el que puede tener una cierta similitud con la tendencia a evitar la motivación y la implicación del estudiante en el aprendizaje, tratando en todo momento de esforzarse lo menos posible.

Es posible que los alumnos del grupo ambivalente, que muestran los niveles de rendimiento académico significativamente más altos que los estudiantes cuyo enfoque es profundo, estén condicionados por los efectos contrapuestos de los dos componentes motivacionales: motivo de logro y miedo al fracaso ambos integrantes del enfoque superficial. En este grupo las mediciones son bajas con un predominio de la motivación superficial. Prácticamente no presenta diferencias con el grupo que adopta predominantemente un enfoque superficial. Llamativamente, se ubican en este grupo los alumnos con las notas promedio más altas.

Al cruzar los valores de las variables relativas a la motivación y al uso de estrategias se puede señalar que no siempre estos alumnos que tienen niveles de motivación más altos también hacen uso de las estrategias en un nivel de mayor complejidad. A su vez, los sujetos con motivación baja tienden a recurrir en mayor medida al uso de estrategias de aprendizaje. Es decir que, si bien estos alumnos no están 
demasiado motivados, igualmente ponen en marcha diversas estrategias cuando aprenden. En los tres perfiles encontramos que las subescalas estratégicas presentan índices más altos que las motivacionales.

Creemos que por ser el cuestionario CEPEA un instrumento diseñado para evaluar enfoques de aprendizaje en estudiantes universitarios australianos, adaptado en forma experimental a alumnos españoles, estaríamos detectando diferencias culturales. Si analizamos las categorías, algunas se encuentran claramente cruzadas; es posible identificar diferencias conceptuales en los ítems correspondientes a las motivaciones superficiales y de logro. Es decir que lo que para los autores del cuestionario representa motivación de logro, para nuestra idiosincrasia corresponde a motivación superficial y viceversa.

En términos generales, los resultados de nuestro trabajo difieren parcialmente de los obtenidos en otros estudios, seguramente debido a particularidades en las características culturales de las distintas poblaciones universitarias. Los valores medios de las mediciones tanto de motivaciones como de estrategias de logro, en general, son llamativamente bajos. Podemos afirmar que en nuestro contexto cultural lo que aparece como 'superficial' realmente es logro. Esto también explicaría un valor sorprendentemente alto de motivaciones y estrategias superficiales en alumnos de alto rendimiento académico.

Si bien es habitual que los estudiantes con enfoque profundo tengan mayores expectativas de éxito y obtengan buenos resultados académicos, hemos comprobado la falta de correlación entre el enfoque profundo y las calificaciones obtenidas por los estudiantes que lo presentan. Los alumnos que adoptan un enfoque profundo no generan, necesariamente, buenos resultados académicos y viceversa. Paradójicamente los niveles de rendimiento académico de estos alumnos no son más altos que el resto de los grupos.

Los alumnos exitosos, aplazados reincidentes, y rezagados, se ubican en los tres grupos (superficial, profundo y ambivalente). A su vez, los alumnos con las notas promedio más altas se ubican en el grupo ambivalente. Es posible que estos últimos estén condicionados por los efectos contrapuestos de los dos componentes motivacionales: motivo de logro y miedo al fracaso.

El alumno opta por un modo u otro de procesar la información, aunque es posible distinguir una determinada dirección evolutiva: a medida que se avanza en la carrera crece la población que adopta el enfoque profundo, lo que indicaría un tránsito en el estilo de aprendizaje de superficial a profundo que podría explicarse como el intento de ajustarse a las demandas percibidas y como una mejora en sus estrategias de aprendizaje. Esto evidenciaría un proceso de mayor adaptación al ambiente universitario (aprende el 'oficio' de estudiante universitario y se acomoda mejor a las reglas).

\section{REFERENCIAS BIBLIOGRÁFICAS}

Barca lozano, A. (1999). Manual del Cuestionario de Procesos de Estudio y Aprendizaje (CEPEA). A Coruña: Revista Galego-Portuguesa de Psicoloxía e educación.

BIGGS, J.B. (1984). Learning strategies, student motivation patterns and subjectively perceived success. En J. R. Kirby (Ed.). cognitive strategies and educational performance (p. 111-134). Orlando, FL. Acad. Press. 
BIGGS, J.B. (1987c). Study Process Questionnaire (SPQ). Hawthorn, Victoria: Australian Council for Educational Research.

ENTWISTLE, N.J. (1988). Motivational factors in student's approaches to learning. En R.R.Schmeck (Ed.), learning strategies and learning styles. Nueva Yorkl: Plenum Press.

FRANSSON, A. (1977). On qualitative differences in learning IV. Effects of motivation and test anxiety on process and outcome. British Journal of Educational Psychology, 47, 244-257.

González Cabanach, R., Barca, A., Valle, A., Porto Rioboo, A. y LemA, S. (1993). El punto de vista del alumno acerca del aprendizaje: Las aproximaciones al aprendizaje y las orientaciones al estudio. En F. Vicente Castro (ed.), Psicología de la educación y del desarrollo. Vol. 2. Badajoz: Psicoex.

González Cabanach, R.; Valle, A. y VÁzquez Grobas, A. (1994). Las estrategias del aprendizaje. En R. González Cabanach: Psicología de la Instrucción. El profesor y el estudiante, La Coruña, Servicio de Publicaciones de la Universidad de La Coruña.

GonZÁLEZ, R. VALLE, A. y col. (1999). Variables Motivacionales, Estrategias de Aprendizaje y Rendimiento Académico en Estudiantes Universitarios. Un modelo de relaciones causales. Rev. de Orientación y Psicopedagogía. Universidad de La Coruña. № 16.

PORTO RIOBOO, A. (1994b). Los enfoques de aprendizaje en contextos educativos: Una aproximación conceptual. En A. Barca, R. González Cabanach, J.L. Marcos, A. Porto Rioboo y A. Valle, Procesos básicos de aprendizaje y aprendizaje escolar. La Coruña: Servicio de Publicaciones de la Universidad de La Coruña.

VALLE ARIAS, A. et. al (1999). Un modelo cognitivo -motivacional explicativo del Rendimiento académico en la universidad. Revista Estudios de Psicología Vol. 63 № 2:77-100.

Valle Arias, A., Barca, A., González Cabanach, R., Porto Rioboo, A. y Santorum, R. (1993). Aprendizaje significativo y enfoques de aprendizaje: El papel del alumno en el proceso de construcción de conocimientos. Revista de Ciencias de la Educación, 156, 481-502.

Valle Arias, A., BARCA, A., GonZÁlez, R y NúÑEZ, J. C. (1995). Las estrategias de aprendizaje: una aproximación teórica y conceptual. Rev. Gallega de Psicopedagoxia. 12:31-58.

Valle Arias, A., González Cabanach, R., Vieiro IGlesias, P. (1997). Características Diferenciales de los enfoques de Aprendizaje en estudiantes universitarios. Rev. de Psicodidáctica № 4. 